\title{
Contextual factors that heighten interest in coalitional alliances with men possessing formidable facial structures
}

\author{
Mitch Brown $^{\mathrm{a}, *}$, Donald F. Sacco ${ }^{\mathrm{b}}$, Nicole Barbaro ${ }^{\mathrm{c}}$, Kelsey M. Drea ${ }^{\mathrm{b}}$ \\ a University of Arkansas, United States of America \\ ${ }^{\mathrm{b}}$ The University of Southern Mississippi, United States of America \\ ${ }^{c}$ Utah Valley University, United States of America
}

A R T I C L E I N F O

\section{Keywords:}

Facial width-to-height ratio

Formidability

Coalition

Combat

Aggression

\begin{abstract}
A B S T R A C T
Individuals use facial width-to-height ratio (fWHR) to infer men's formidability. We hypothesized that fWHR assessments would form a basis for men's coalitional value, with high-fWHR men being valuable in roles requiring physical strength. Five studies $(N=1323)$ tested how perceptions of formidability influence coalitional decisions. In addition to replicating previous findings indicating a preference for high-fWHR men in tasks requiring strength (Study 1), the formidability inference most associated with this high-fWHR preference was perceived strength and not aggressiveness (Studies 2a, 2b). Two pre-registered studies showed that activating competitive motivations increased preferences for high-fWHR allies (Study 3), though this preference appeared driven by a tolerance for high-fWHR men rather than an interest (Study 4). Findings provide evidence for how inferences of fWHR shape interpersonal preferences based on social contexts.
\end{abstract}

Despite sharp declines in recent history, physical violence remains pervasive across human societies. This violence has exerted significant selection pressure for adaptations that manage the costs and benefits of aggression. Adaptations include the quick recognition of formidable physical features to estimate the capability of others to inflict harm on the perceiver (Sell, Tooby, \& Cosmides, 2009) and perceptual systems to detect threats (Neuberg, Kenrick, \& Schaller, 2011). Humans infer formidability through physical cues indicating upper body strength, particularly men's due to their greater proclivity toward physical conflict throughout evolutionary history (Lukaszewski, Simmons, Anderson, \& Roney, 2016; Puts, 2010; Sell, Hone, \& Pound, 2012). Although bodily cues provide the most accurate information, humans also rely heavily on facial features due to the immediacy of face-to-face contact. Facial features allow individuals to estimate men's aggressive tendencies and thus their capability to inflict harm. Perceivers could then estimate the social benefits (e.g., protection) and costs (e.g., physical harm) of formidable men for group living based on inferences from facial cues (Zebrowitz \& Montepare, 2006).

One facial characteristic that humans use to infer formidability is men's facial width-to-height ratio (fWHR), the ratio of bizygomatic width relative to upper face height. Men with a higher ratio (high-fWHR) are perceived as aggressive and actually are more aggressive than lowfWHR men (Caton, Pearson, \& Dixson, 2022; Carré, McCormick, \& Mondloch, 2009; Durkee \& Ayers, 2021; Geniole \& McCormick, 2015; Goetz et al., 2013; Haselhuhn, Ormiston, \& Wong, 2015; Welker, Goetz, Galicia, Liphardt, \& Carré, 2015; cf. Deaner, Goetz, Shattuck, \& Schnotala, 2012). Despite their potential costs, high-fWHR men could benefit the group by offering protection. The current research considered how men's fWHR shapes coalitional preferences based on the trade-off of high-fWHR men being beneficial in group combat despite the risk of physical harm they pose.

\section{Features associated with formidability}

Inferences of male formidability appear multimodal, with perceivers relying on various behaviors and physical features. Behaviors include head-tilting (Toscano, Schubert, \& Giessner, 2018) and lowered vocal pitch (Aung, Rosenfield, \& Puts, 2021), which are hypothesized to increase perceptions of men's prowess. However, behavioral displays may only create an intimidating display without reflecting actual ability. The difficulty in modifying facial structures implicates them as useful for estimating formidability in social targets accurately. Masculinized

\footnotetext{
Data and materials from all studies, including pre-registration plans for Studies 3-4, are available: https://osf.io/gcpk6/?view_only = 88914a8be39d441ba83a83 $82 \mathrm{f} 923 \mathrm{~d} 821$

* Corresponding author at: Memorial Hall 202G, Department of Psychological Science, University of Arkansas, Fayetteville AR 72701, United States of America. E-mail address: mb103@uark.edu (M. Brown).
} 
facial features provide such information for men (Caton, Zhao, Lewis, \& Dixson, 2022). Masculinization is rooted in fetal androgen exposure and pubertal testosterone surges, which foster muscle growth and face widening (Griggs et al., 1989; Whitehouse et al., 2015). The resulting upper body strength is associated with masculinized facial features from which perceivers can infer men's actual strength (Holzleitner \& Perrett, 2016; Price, Sheehy-Skeffington, Sidanius, \& Pound, 2017).

Men's fWHR appears to contribute to perceptions of formidability. Though not exclusively sexually dimorphic (e.g., Lefevre et al., 2012), several components of fWHR are diagnostic of masculinization in men. Men have wider and longer lower faces which are associated with androgen activity throughout development (Hodges-Simeon et al., 2021; Hodges-Simeon, Hanson Sobraske, Samore, Gurven, \& Gaulin, 2016). In fact, recent findings suggest that facial width is sexually dimorphic, with the connotation of threat through fWHR being amplified by the height of men's faces (Caton \& Dixson, 2022; Kajonius \& Eldblom, 2020; Liu, Wen, \& Zheng, 2022). Male formidability appears to have been selected due to recurrent physical conflict and intrasexual competition (Puts, 2010), implicating components of fWHR as a result of sexual selection. This selection could have led to the emergence of functional heuristics for men's formidability when these features are presented together (Dixson, 2018). The threatening appearance of high-fWHR men could lead them to perceive themselves as advantaged in conflict and capable of using aggressive strategies (Haselhuhn, Wong, \& Ormiston, 2013; Mileva, Cowan, Cobey, Knowles, \& Little, 2014).

Several heuristics emerge readily through fWHR. For example, highfWHR men are heuristically associated with toughness (Brown, Bauer, Sacco, \& Capron, 2021; Deska et al., n.d.; Deska \& Hugenberg, 2018). Although some of these inferences appear rooted in stereotypes of aggression (Matsumoto \& Hwang, 2021), other judgments have a kernel of truth. High-fWHR men have more favorable win-loss records in mixed martial arts, particularly when considering grappling capabilities (Caton, Hannan, \& Dixson, 2022; Třebický et al., 2015; Zilioli et al., 2015). Forensic records of male skeletons analyzed to determine causes of death (e.g., homicide) further indicate that high-fWHR men are historically less likely to die from violent encounters (Stirrat, Stulp, \& Pollet, 2012). This aggression among formidable men is further exemplified in small-scale societies (Christiansen \& Winkler, 1992; von Rueden \& Van Vugt, 2015). Formidability inferences via fWHR could serve to identify men's aggressive intent and physical advantages.

\section{Coalitional value of formidability}

Formidability inferences could inform perceivers of men's costs and benefits for specific coalitional roles. Regarding costs, high-fWHR men appear threatening (Geniole, Denson, Dixson, Carré, \& McCormick, 2015), intimidating (Hehman, Leitner, \& Gaertner, 2013), and prone to bullying (Brown, Tracy, \& Boykin, 2022). These perceptions foster desires for social distance from high-fWHR men (Lieberz et al., 2017). Nonetheless, formidable men afford benefits to group living. Individuals prefer formidable men for protection cross-culturally (Apicella, 2014; Brown, Donahoe, \& Boykin, 2022; Lukaszewski et al., 2016; von Rueden \& Van Vugt, 2015). High-fWHR men are seen as good fighters (Brown, Sacco, \& Barbaro, 2022; Zilioli et al., 2015) and desirable for coalitions that benefit from strength (e.g., protection; Hehman, Leitner, Deegan, \& Gaertner, 2015). Taken together, in certain contexts, individuals may be motivated to ally themselves with high-fWHR men as the protection against hostile outgroups they afford may outweigh concerns regarding their potential for ingroup exploitation or harm.

Nonetheless, the use of fWHR in affordance judgments presents limitations. Several trait inferences common in WEIRD cultures (e.g., dominance) are not cross-culturally robust (Durkee \& Ayers, 2021) or manifest differently (Magginetti, 2015). In certain ecologies where the costs of formidable group members could be particularly salient, masculinized facial structures are aversive (Borras-Guevara, Batres, \& Perrett,
2017; Brooks et al., 2011; DeBruine, Jones, Little, Crawford, \& Welling, 2011). Previous research indicates that the aggressiveness inferred in masculine features impedes their desirability, which could lead individuals to be averse to high-fWHR men in contexts where aggression has considerable costs (Geniole \& McCormick, 2013). Human perceptual systems appear to identify formidability through fWHR for perceivers to consider whether the benefits of formidability outweigh the costs in a given context. Aggressive tendencies are cross-culturally inferred through men's fWHR, which may position research to focus on physical abilities inferred through fWHR (Deska, Lloyd, \& Hugenberg, 2018a; Durkee \& Ayers, 2021).

\section{Current research}

The current research examined the trade-off that perceivers of men's fWHR could consider when deciding whether to affiliate with formidable men and for what types of roles. We first replicated previous findings demonstrating individuals prefer high-fWHR men for tasks that require strength using an expanded stimulus set and larger sample (Study 1 ). We next assessed the perceptual bases for the preference of highfWHR men through trait inferences related to physical and mental capabilities (Studies 2a and 2b). Given the different value of high- and lowfWHR men in competitive and cooperative roles, we investigated next motives underlying these preferences by priming competitive and cooperative motives before participants indicated their preferences for highand low-fWHR men (Study 3). Finally, we considered whether preferences for high-fWHR men are rooted in motivations to approach allies or whether these preferences represent a tolerance to high-fWHR men by using a visual bias task (Study 4). Data in each study were collected in single waves and not analyzed until completion.

\section{Study 1}

Individuals prefer high-fWHR men as teammates to complete tasks requiring strength (Hehman et al., 2015). Although theoretically sensible, results from previous studies are limited by stimulus choices and small samples. This conceptual replication addresses these limitations with an expanded number of male stimuli and a larger sample. We also tasked participants to indicate preferences for high- and low-fWHR men for group roles that are less variable in their needs than what has been used in previous research. For example, previous research has used American football as a "strength" task (Hehman et al., 2015), but players in this sport are highly specialized for their position and some positions are less reliant on strength. The strength task in this study was tugof-war because it requires strength among all members of a team for success. The cooperative task assumed irrelevant to physical strength was an escape room. We predicted that high-fWHR men will be preferred in tug-of-war but not preferred for the escape room.

Although men and women are both adept at inferring formidability, men's historically greater involvement in physical conflict could position them to prefer high-fWHR allies more readily in their coalitions (McDonald, Navarrete, \& Van Vugt, 2012). Conversely, the dimorphism in physical size between men and women could implicate women as more vulnerable to exploitation by formidable men, resulting in a greater overall aversion to affiliate with high-fWHR men. We predicted that this preference for high-fWHR men would therefore be larger for men.

\subsection{Method}

\subsubsection{Participants}

We recruited 199 U.S. participants through Mechanical Turk for $\$ 0.35$ (US). Three participants were excluded for not completing the full study $\left(n_{\text {Final }}=195 ; 84\right.$ men, 111 women; $M_{\text {Age }}=36.63$ years, $S D=13.22 ; 64.1 \%$ White, $14.9 \%$ Asian, 9.2\% Black, $8.2 \%$ Latin, $7 \%$ 
Other). A sensitivity analysis indicated we had adequate power for small effects (Cohen's $f=0.10,1-\beta=0.80$ ).

\subsubsection{Materials and procedure}

4.1.2.1. Target faces. Participants built coalitions based on arrays of 20 neutrally expressive, color-presented White male faces from the Chicago Faces Database (Ma, Correll, \& Wittenbrink, 2015; Fig. 1). Faces naturally varied in fWHR. We used the 10 highest and 10 lowest fWHRs in the set (Deska \& Hugenberg, 2018). The difference between stimulus categories was large $(d=6.32)$, allowing us to compare relative differences as high-fWHR (i.e., formidable) and low-fWHR (i.e., non-formidable) categories, akin to how humans naturally categorize others (see Phillips, Slepian, \& Hughes, 2018).

Norming data indicated that these categories did not differ in attractiveness $(d=-0.05)$. Although previous findings demonstrated a lack predictive value of attractiveness for coalition-building (Lukaszewski et al., 2016), similar levels of attractiveness would minimize possible halo effects and reduce concerns of high-fWHR men being perceived as less attractive (Dixson, Lee, Sherlock, \& Talamas, 2017). Two manipulation check items assessed the extent targets appeared strong and good at fighting on 7-point scales ( $1=$ Not at All; $7=$ Very $M u c h)$. Items were highly correlated in both target categories and aggregated as composite formidability scores $(r s>0.82)$.

4.1.2.2. Coalition-building. We presented faces in two arrays of 10 . Participants chose between zero and five target individuals for two hypothetical cooperative tasks (e.g., Brown, Sacco, \& Drea, 2022). One task required choosing allies for tug-of-war, a task requiring strength. The other task was choosing allies for an escape room, ostensibly irrelevant to strength. Participants viewed arrays in random, counterbalanced orders (i.e., some viewed a set for tug-of-war first and others for the escape room first). Five high- and five low-fWHR targets appeared in each array. We summed the high- and low-fWHR target participants chosen for both tasks.

\subsection{Results}

\subsubsection{Formidability}

Participants perceived high-fWHR targets as more formidable $(M=4.81, S D=0.80)$ than low-fWHR targets $(M=3.94$, $S D=1.02), t(194)=15.57, p<0.001, d=1.11$.

\subsubsection{Coalition-building}

We conducted a 2 (participant sex: male vs. female) $\times 2$ (target fWHR: high vs. low) $\times 2$ (task: tug-of-war vs. escape room) mixedmodel ANOVA with repeated factors for target fWHR and task. We decomposed significant interactions in all studies using simple effects tests. A target fWHR main effect indicated participants selected more high-fWHR targets $(M=2.56, S D=1.08)$ than low-fWHR targets $(M=2.09, S D=1.01), F(1,193)=25.95, p<0.001, \eta_{p}^{2}=0.119$.

A target fWHR $\times$ task interaction emerged, $F(1,193)=102.55$, $p<0.001, \eta_{p}{ }^{2}=0.347$ (Fig. 2). Participants selected more high-fWHR targets for tug-of-war $(M=3.10, S D=1.15)$ than low-fWHR targets $(M=1.58, S D=0.94), F(1,193)=119.14, p<0.001, \eta_{p}{ }^{2}=0.382$.
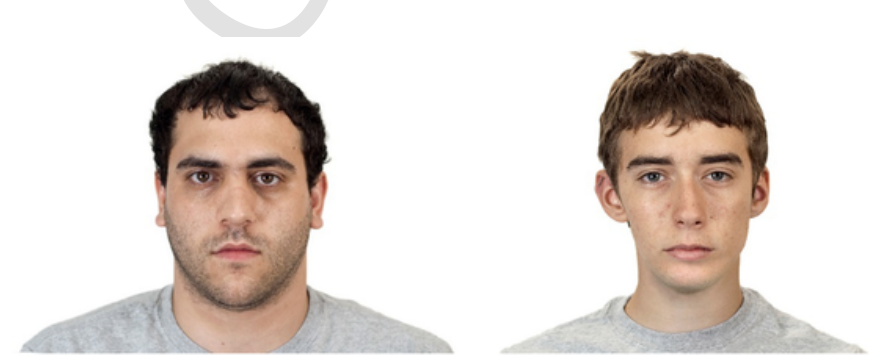

Fig. 1. Examples of relatively high- (left) and low-fWHR targets (right).

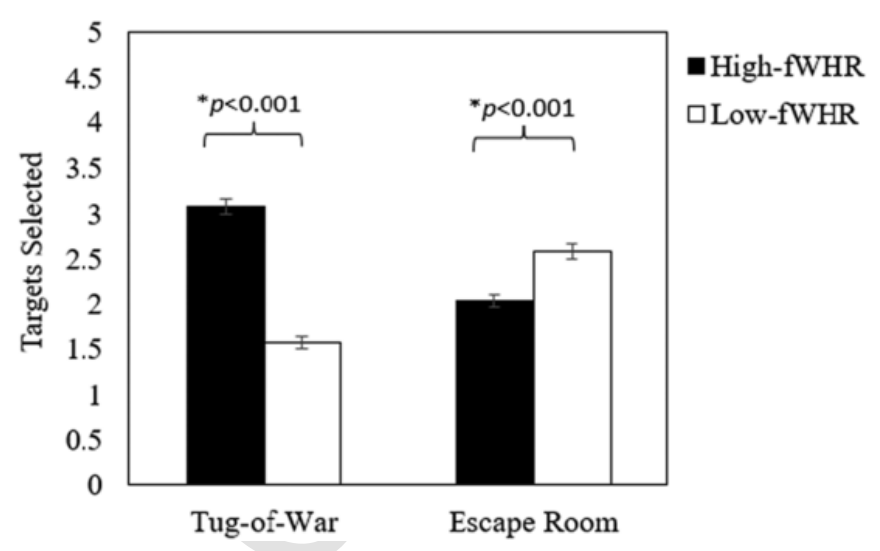

Fig. 2. Selection of high- and low-fWHR targets for tug of war and escape room tasks in Study 1 (with standard errors).

Conversely, participants selected more low-fWHR targets for escape rooms $(M=2.61, S D=1.09)$ than high-fWHR targets $(M=2.03$, $S D=1.02), F(1,193)=17.50, p<0.001, \eta_{p}{ }^{2}=0.083$. No other main effects or interactions emerged, $\mathrm{Fs}<2.68$, $p \mathrm{~s}>0.102 .{ }^{1}$

\subsection{Discussion}

Participants preferred high-fWHR men for tug-of-war. High-fWHR men appeared to have been preferred for tug-of-war presumably due to being perceived as strong. Affordance judgments of high-fWHR men's capabilities could motivate an interest in preferring formidable allies for strength tasks. Conversely, low-fWHR targets were preferred for escape rooms, presumably this preference reflects perceptions of highfWHR men as costly in strength-irrelevant domains. Study 2a sought to identify potential perceptual bases of these preferences.

No difference emerged in preference between men and women. Women's preferences could represent an interest in optimizing protection against threats (Snyder et al., 2011), whereas men's preferences could reflect choosing coalitional allies. Given the null sex-differences, we did not consider sex-differences in subsequent studies.

\section{Study 2a}

This study assessed the bases of preferences for high- and low-fWHR men through inferences of strength and cooperativeness. We predicted the strength advantage of high-fWHR targets would be a basis for a tugof-war preference. Conversely, the possibility that escape rooms require cooperation led us to assess perceived cooperativeness. We predicted that the cooperation advantage for low-fWHR targets would be a basis of choosing these targets for escape rooms (Haselhuhn et al., 2013).

\subsection{Method}

\subsubsection{Participants}

We recruited 200 undergraduates from a large public university in Northwest Arkansas for course credit. We excluded one participant for not responding to the coalition-building task $\left(n_{\text {Final }}=199 ; 145\right.$ women, 50 men, 4 undisclosed; $M_{\text {Age }}=18.98$ years, $S D=2.42$; $80.4 \%$ White, 7.5\% Latin, 5.5\% Asian, 2.5\% Black, 3.5\% Other). A sensitivity analysis indicated adequate power to detect small effects $(f=0.10,1$ $\beta=0.80$ ).

\footnotetext{
1 An analysis considering order or counterbalance did not meaningfully change results.
} 


\subsubsection{Materials and procedure}

We used the same task as Study 1 in a between-subjects capacity. That is, participants chose up to five targets for either a tug-of-war ( $n=100)$ or an escape room $(n=99)$. Participants responded to the array from Study 1 that elicited the largest difference in formidability perceptions $(d=7.47)$. Participants rated targets along single 7-point scales for perceived strength and cooperativeness $(1=$ Not at All; 7 = Very Much).

\subsection{Results}

\subsubsection{Affordance judgments}

Participants perceived the high-fWHR targets as stronger $(M=4.90, S D=0.79)$ than low-fWHR targets $(M=2.92$, $S D=0.71), t(198)=34.65, p<0.001, d=2.45$. Low-fWHR targets were perceived as more cooperative $(M=4.26, S D=0.84)$ than highfWHR targets $(M=3.66, S D=0.82), t(198)=9.11, p<0.001$, $d=0.64$.

\subsubsection{Coalition-building}

We used a 2 (condition: tug of war vs. escape room) $\times 2$ (target fWHR: high-fWHR vs. low-fWHR) mixed-model ANOVA with repeated factors over Target fWHR. A target fWHR main effect indicated that participants chose high-fWHR targets more often $(M=2.81$, $S D=1.41)$ than low-fWHR targets $(M=1.75, S D=1.21), F(1$, $197)=82.92, p<0.001, \eta_{p}^{2}=0.296$. A condition main effect indicated participants chose more targets for tug-of-war $(M=2.41$, $S D=0.79)$ than escape rooms $(M=2.16, S D=1.01), F(1$, 197) $=17.98, p<0.001, \eta_{p}^{2}=0.084$.

A condition $\times$ target fWHR emerged, $F(1,197)=262.31$, $p<0.001, \eta_{p}{ }^{2}=0.571$ (Fig. 3). Participants selected more high-fWHR targets for tug-of-war $(M=3.87, S D=0.89)$ than low-fWHR targets $(M=0.95, S D=0.70), F(1,197)=321.73, p<0.001, \eta_{p}{ }^{2}=0.620$. Participants chose more low-fWHR targets for an escape room $(M=2.57, S D=1.06)$ than high-fWHR targets $(M=1.75$, $S D=0.97), F(1,197)=25.00, p<0.001, \eta_{p}{ }^{2}=0.113$.

\subsubsection{Bases of selection}

Our next step was understanding the basis of choosing allies through perceived strength and cooperativeness. The between-subjects nature of our task necessitated two separate zero-order correlations for both conditions. We calculated difference scores for target categories for choices, perceived strength, and perceived cooperativeness. Higher scores reflected an advantage for high-fWHR targets. Unlike difference scores for between-subjects designs, within-subjects variables are not impeded by unreliability and are therefore amenable to difference score analyses (Thomas \& Zumbo, 2012; Trafimow, 2015).

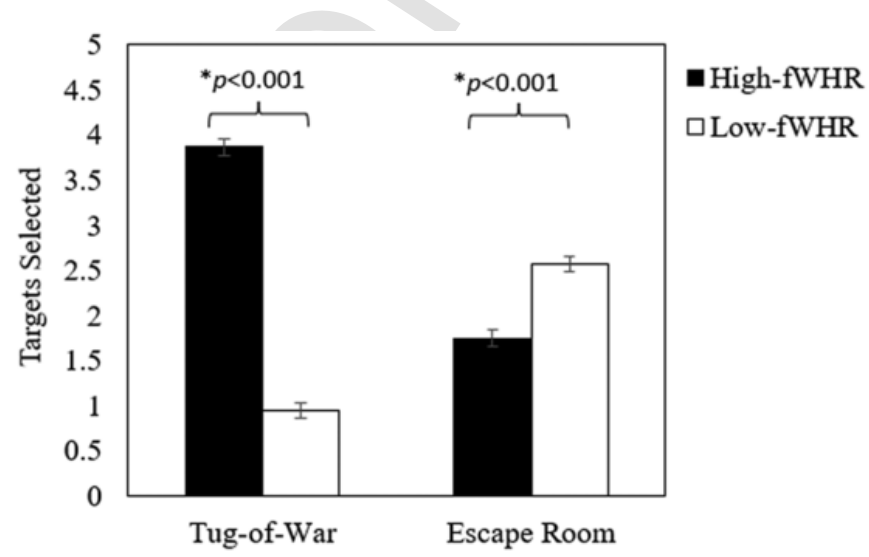

Fig. 3. Selection of high- and low-fWHR targets for tug of war and escape room tasks in Study 2a (with standard errors).
The perceived strength advantage of high-fWHR targets was associated with choosing high-fWHR men for tug-of-war $(r=0.23$, $p=0.021)$. Perceived cooperation was not associated with tug-of-war selections $(r=0.02, p=0.820)$. No association emerged for perceived strength and escape room decisions $(r=0.10, p=0.321)$. Unexpectedly, a perceived cooperation advantage of high-fWHR targets was associated with choosing them for the escape room $(r=0.22$, $p=0.028$ ).

\subsection{Discussion}

We replicated results from Study 1 by demonstrating a preference for high-fWHR men in strength tasks, further indicating that this preference is based in perceiving high-fWHR men as stronger. Interestingly, perceptions of high-fWHR men as uncooperative were not the basis of an aversion to them in escape rooms. That is, perceptions of high-fWHR men as cooperative were associated with a preference for high-fWHR men in escape rooms. This association could suggest participants saw high-fWHR men as able to provide unique benefits for the task (e.g., group rule enforcement; Lukaszewski et al., 2016). Nonetheless, the basis of the aversion to high-fWHR men remains unclear. Study $2 \mathrm{~b}$ considered additional judgments to identify the basis of the high-fWHR aversion in escape rooms.

\section{Study $2 b$}

Strength and anger inferences frequently covary with high- and lowfWHR in men (MacDonell, Geniole, \& McCormick, 2018), suggesting potential ambiguities in understanding whether perceived strength or aggressiveness shapes coalitional decisions with high-fWHR men. Study $2 \mathrm{~b}$ sought to clarify whether preferences for high-fWHR men in tug-ofwar are driven more by inferences of strength or aggressiveness.

Despite high-fWHR targets being perceived as uncooperative, results from Study 2a indicated that perceptions of high-fWHR men as cooperative facilitated preferences for them in escape rooms. This finding could suggest that inferences of cooperativeness through fWHR are less robust than those traits related to formidability. Another purpose of Study $2 \mathrm{~b}$ was to identify additional affordance judgments of fWHR. High-fWHR men are frequently perceived as lacking mental complexity (e.g., intelligence; Brown, Bauer, et al., 2021; Deska, Lloyd, \& Hugenberg, 2018b), a stereotype without any kernel of truth (Kosinski, 2017). This stereotype could foster an aversion to high-fWHR men in escape rooms, given that these tasks often require creativity and intelligence that perceivers assume high-fWHR men lack. We thus assessed perceived intelligence and creativity of high- and low-fWHR men in this study.

We also considered participants' additional perceptions of high-and low-fWHR men being generally effective for a given task. This allowed us to identify complementary bases of coalitional decisions for high- and low-fWHR men. We predicted that perceived effectiveness for tug-ofwar for high-fWHR men would be a basis for choosing them in that task and perceived effectiveness for low-fWHR for escape rooms as a basis for choosing them in escape rooms.

\subsection{Method}

\subsubsection{Participants}

A sample of 170 undergraduates from a public university in Northwest Arkansas completed this study for course credit (126 women, 42 men, 2 identifying as neither; $M_{\text {Age }}=18.66$ years, $S D=1.49 ; 78.2 \%$ White, $7.1 \%$ Black, 6.5\% Latin, 2.4\% Asian, 5.3\% Other). No data were excluded. Sensitivity analyses indicated we had sufficient power to detect small effects $(f=0.11,1-\beta=0.80)$. 


\subsubsection{Materials and procedure}

This study employed the same methods as Study 2a, with participants being assigned to the tug-of-war $(n=86)$ or escape room condition $(n=84)$. In addition to the same preference task and assessments for strength and cooperativeness, we assessed perceptions of intelligence (i.e., a single-item corollary to mental sophistication), creativity, and aggressiveness. A final item assessed how effective a target would be for the task to which participants were assigned. All items used the same 7-point scales as Study 2a.

\subsection{Results}

\subsubsection{Affordance judgments}

High-fWHR targets were perceived as stronger and more aggressive than low-fWHR targets. Low-fWHR targets were perceived as more creative, cooperative, and intelligent. Table 1 provides relevant statistics.

\subsubsection{Coalition-building}

We used a 2 (condition: tug of war vs. escape room) $\times 2$ (target fWHR: high-fWHR vs. low-fWHR) mixed-model ANOVA with repeated factors over target fWHR. A target fWHR main effect indicated participants chose more high-fWHR targets $(M=2.67, S D=1.34)$ than lowfWHR targets $(M=1.77, S D=1.12), F(1,168)=61.53, p<0.001$, $\eta_{p}{ }^{2}=0.268$. A condition main effect indicated participants chose more targets for tug-of-war $(M=2.35, S D=0.77)$ than escape rooms $(M=2.17, S D=1.02), F(1,168)=8.25, p=0.005, \eta_{p}^{2}=0.047$.

A condition $\times$ target fWHR emerged, $F(1,168)=183.13$, $p<0.001, \eta_{p}{ }^{2}=0.522$ (Fig. 4). Participants chose more high-fWHR targets for tug-of-war $(M=3.67, S D=0.82)$ than low-fWHR targets $(M=1.03, S D=0.71), F(1,168)=231.20, p<0.001, \eta_{p}{ }^{2}=0.579$. Low-fWHR targets were chosen more for escape rooms $(M=2.52$, $S D=0.95)$ than high-fWHR targets $(M=1.82, S D=1.09), F(1$, 168) $=15.99, p<0.001, \eta_{p}^{2}=0.087$.

\subsubsection{Bases of selection}

We used the zero-order correlation strategy from Study 2a. The perceived strength advantage of high-fWHR targets was associated with

Table 1

Mean perceptions (and SD) for high- and low-fWHR targets in Study 2b.

\begin{tabular}{lllll}
\hline & High-fWHR & Low-fWHR & $t$ & $d$ \\
\hline Strength & $5.09(0.70)$ & $3.05(0.71)$ & $32.64_{*}$ & 2.50 \\
Aggressiveness & $4.46(1.00)$ & $3.01(0.85)$ & $18.48_{*}$ & 1.41 \\
Creativity & $3.29(0.72)$ & $3.96(0.81)$ & $-10.79_{*}$ & 0.83 \\
Cooperativeness & $3.74(0.76)$ & $4.34(0.77)$ & $-9.33_{*}$ & 0.71 \\
Intelligence & $3.73(0.79)$ & $4.47(0.81)$ & $-12.19_{*}$ & 0.93 \\
\hline
\end{tabular}

$* p<0.001$.

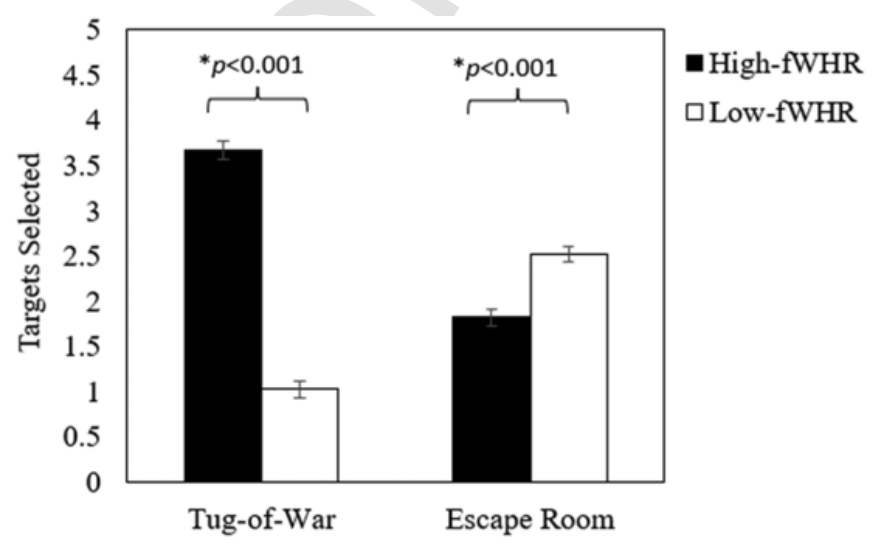

Fig. 4. Selection of high- and low-fWHR targets for tug of war and escape room tasks in Study $2 \mathrm{~b}$ (with standard errors). choosing them more frequently for tug-of-war. No other associations emerged for tug-of-war. For escape rooms, two unexpected correlations emerged: Perceptions of high-fWHR men as creative were unexpectedly correlated with choosing them more frequently (Table 2).

\subsubsection{Effectiveness}

We used a similarly dimensioned 2-way ANOVA to assess general effectiveness in the given task. A target fWHR main effect indicated highfWHR targets $(M=4.58, S D=0.95)$ were perceived as more effective in their given task than were low-fWHR targets $(M=4.03$, $S D=0.90), F(1,168)=52.66, p<0.001, \eta_{p}{ }^{2}=0.239$. No Condition main effect emerged, $F(1,168)=1.00, p=0.318, \eta_{p}^{2}=0.006$.

A Condition $\times$ Target fWHR interaction emerged, $F(1$, $168)=169.35, p<0.001, \eta_{p}{ }^{2}=0.502$. High-fWHR targets were perceived as more effective in tug-of-war $(M=5.00, S D=0.88)$ than low-fWHR targets $(M=3.51, S D=0.74), F(1,168)=207.88$, $p<0.001, \eta_{p}{ }^{2}=0.553$. Low-fWHR targets were perceived as more effective in escape rooms $(M=4.57, S D=0.74)$ than high-fWHR targets $(M=4.14, S D=0.83), F(1,168)=16.38, p<0.001$, $\eta_{p}^{2}=0.089$.

\subsubsection{Bases of selection}

We conducted two mediation analyses considering Condition as the predictor, number of high- and low-fWHR targets chosen as outcomes, and corresponding perceptions of general effectiveness for high- and low-fWHR targets for the given task as mediators. We employed Model 4 of PROCESS using 5000 bootstraps (Hayes, 2013). The choice of highfWHR targets for tug-of-war was mediated by perceiving high-fWHR targets as generally more effective for tug-of-war, 95\% CI $[-0.32$, -0.02]. Choosing low-fWHR for an escape room was also mediated by perceptions of low-fWHR men's general effectiveness in escape rooms, $95 \%$ CI $[0.18,0.59]$.

\subsection{Discussion}

Preferences for high-fWHR men in tug-of-war were based in strength, but not aggressiveness. This finding complements previous work by demonstrating how multifaceted formidability assessments are (Durkee \& Ayers, 2021). Perceived cooperativeness was unassociated with choosing high- or low-fWHR men, suggesting the previously identified association is less reliable due to greater idiosyncrasies in understanding perceptions of high-fWHR toward cooperation.

Perceptions of high-fWHR men as creative and intelligent were associated with preferring high-fWHR men for escape rooms. Perceivers could have recognized the targets as sufficiently creative and intelligent for the task. These results are further evidence, when paired with Study 2a, that the connotation of creativity and intelligence are less apparent through fWHR than formidability. This discrepancy could reflect the fact that fWHR is a veridical cue to men's actual formidability (e.g., Zilioli et al., 2015), whereas inferences of high-fWHR men as unintelligent or uncreative are rooted in unfounded stereotypes (Kosinski, 2017).

Along with specific contexts, it could be possible that different motivations similarly shape coalitional decisions. That is, certain motiva-

\section{Table 2}

Bivariate correlations for each difference score in affordance judgments and selection of targets in Study $2 \mathrm{~b}$.

\begin{tabular}{clllll}
\hline & Strength & Aggression & Creativity & Cooperativeness & Intelligence \\
\hline $\begin{array}{c}\text { Tug-of-War } \\
\text { Selection }\end{array}$ & $0.25^{*}$ & -0.02 & 0.11 & 0.15 & 0.08 \\
$\begin{array}{c}\text { Escape Room } \\
\text { Selection }\end{array}$ & 0.02 & -0.14 & $0.26^{*}$ & 0.14 & $0.37^{* *}$ \\
\hline
\end{tabular}

Note. Higher values represent an advantage for high-fWHR targets in perceptions and selection. $* p<0.05, * * p<0.001$. 
tional states could foster a preference allies whose abilities could facilitate group goals akin to those of a given task. Study 3 considered the trade-offs in choosing allies based on motivational states.

\section{Study 3}

Study 3 considered competitive and cooperative motivations. The motivation to protect one's ingroup from threats could lead to choosing men capable of optimizing group interest (Montoya \& Pittinsky, 2013). High-fWHR men afford benefits when competing for finite resources, given inferences of their competitiveness that corresponds with actual competitive intentions (Haselhuhn et al., 2013). We predicted that individuals would build coalitions with more high-fWHR men when competitive motives are salient (pre-registered hypothesis).

Nonetheless, the costs of high-fWHR men could exceed their benefits in tasks that do not require competition. Perceptions of low-fWHR men as cooperative could implicate them as more desirable in contexts that require cooperation more readily without having to consider the trade-offs inherent in choosing high-fWHR men. We predicted that lowfWHR men would be chosen more than high-fWHR men when cooperation motives are salient (pre-registered hypothesis).

\subsection{Method}

\subsubsection{Participants}

A sample of 498 undergraduates from a large public university in Southern Mississippi completed this study for course credit. Based on the pre-registered power analysis, we sought to recruit at least 200 participants to detect small effects $(f=0.10,1-\beta=0.80)$. We oversampled to ensure sufficient power in case of exclusions. Thirteen participants did not respond to the prompt (e.g., writing nonsense, blank textboxes), resulting in their exclusion ( $n_{\text {Final }}=485 ; 379$ women, 104 men, 2 undisclosed; $M_{\text {Age }}=20.35, S D=5.09 ; 59.2 \%$ White, $32.8 \%$ Black, 3.1\% Latin, 2.9\% Asian, 2.1\% Other). ${ }^{2}$

\subsubsection{Materials and procedure}

Participants were randomly assigned to be primed with competitive ( $n=240$ ) or cooperative $(n=245)$ motives (Montoya \& Pittinsky, 2013). To prime competitive motives, participants wrote about how students from their university would treat students from the rival school during a football game. To prime cooperative motives, participants wrote about how students would treat rival students in a university exchange program. Participants then chose up to five individuals to accompany them to a football game at the rival university from the array from Studies $2 \mathrm{a}$ and $2 \mathrm{~b}$ while using the instructions from Study $1 .^{3}$

\subsection{Results}

We used a 2 (condition: competitive vs. cooperative) $\times 2$ (target fWHR: high vs. low) mixed-model ANOVA with repeated factors over the Target fWHR. A target fWHR main effect indicated participants chose more high-fWHR targets for their coalitions $(M=2.44$, $S D=1.09)$ than low-fWHR targets $(M=2.12, S D=1.01), F(1$, 483) $=16.97, p<0.001, \eta_{p}^{2}=0.034$. No condition main effect emerged, $F(1,483)=0.10, p=0.749, \eta_{p}^{2}<0.001$.

A condition $\times$ target fWHR interaction emerged, $F(1,476)=6.55$, $p=0.011, \eta_{p}{ }^{2}=0.013$ (see Fig. 5). Competition-primed participants chose more high-fWHR targets $(M=2.55, S D=1.12)$ than low-fWHR targets $(M=2.01, S D=1.05), F(1,475)=22.07, p<0.001$, $\eta_{p}{ }^{2}=0.044$. Cooperation-primed participants did not differ in choos-

2 Including all participants did not meaningfully alter findings for Studies 3 and 4.

3 We assessed individual differences in group-favoring norms in our preregistration. We provide these nonsignificant results through OSF.

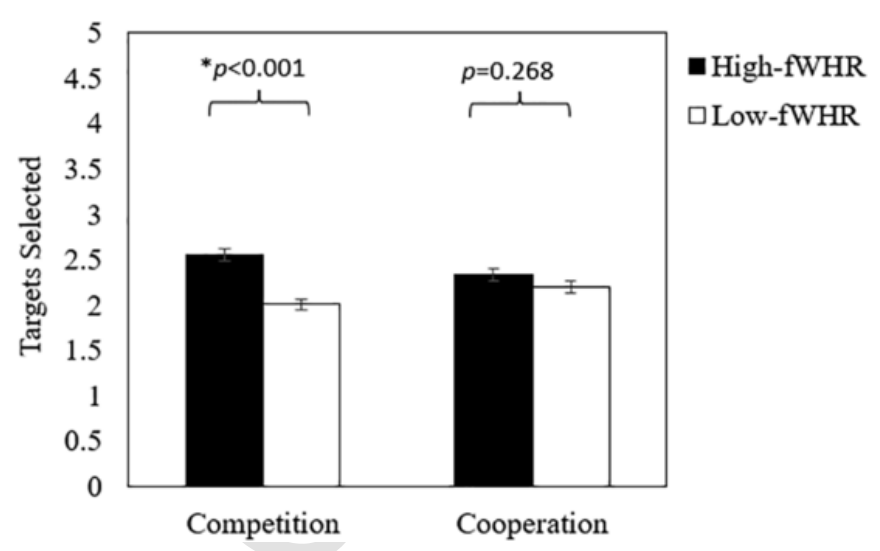

Fig. 5. Selection of high- and low-fWHR targets for Competitive and Cooperative motives in Study 3 (with standard errors).

ing high- $(M=2.33, S D=1.05)$ and low-fWHR targets $(M=2.20$, $S D=0.96), F(1,475)=1.23, p=0.268, \eta_{p}^{2}=0.003$.

\subsection{Discussion}

Competition-primed participants preferred high-fWHR men, presumably to identify strong coalitional allies. However, cooperationprimed participants did not prefer low-fWHR men. Although low-fWHR men could appear cooperative, coalitions could still benefit from the intergroup protection offered by high-fWHR men. These findings provide additional support to Studies $2 \mathrm{a}$ and $2 \mathrm{~b}$ demonstrating a relatively weak and inconsistent connotation of cooperative intent through fWHR. Based on these results, it is unclear whether preferences for high-fWHR men were based in an interest in formidability or a tolerance for risky allies. Study 4 sought to determine the nature of this high-fWHR preference with approach/avoidance responses.

\section{Study 4}

Study 4 examined whether coalitional preferences for high- and low-fWHR men are based in an interest in formidability or tolerance. This study considered the extent to which high- and low-fWHR men elicit approach or avoidance reactions when competitive and cooperative motives are primed. We used a visual bias task that has previously been identified as capable of providing a behavioral proxy for neural activity in cortical areas implicated in approach and avoidance motivations (Miller, Prokosch, \& Maner, 2012), as evidenced by previous electroencephalographic studies (Harmon-Jones \& Allen, 1998). This paradigm has become increasingly popular in identifying associations between motivation and face perception to identify the motivational underpinnings of various preferences without explicit awareness of the purpose of a given study (Brown, Sacco, \& Medlin, 2019; Slepian, Young, \& Harmon-Jones, 2017).

The perceived threat associated with high-fWHR led us to predict individuals will be avoidant toward high-fWHR men independent of motivational state. However, activation of cooperative and competitive motives should modulate responses. We hypothesized that activating competitive motives would downregulate avoidance for coalitionbuilding (preregistered hypothesis), with participants having a reduced aversion toward high-fWHR men. Conversely, we predicted that cooperative motives would heighten avoidance.

\subsection{Method}

\subsubsection{Participants}

A sample of 293 participants in Southern Mississippi completed this study for course credit. We considered only right-handed participants 
(Jewell \& McCourt, 2000). Based on pre-registered power analyses, we recruited at least 200 participants to detect small effects $(f=0.10,1$ $\beta=0.80$ ). We oversampled in case of exclusions. Eight participants were excluded for failing to respond to the prompt per a quality check or not responding to more than two critical trials in the bisection task. This latter criterion ensured a sufficient number of trials to calculate a composite for dichotomous forced-choice tasks (Pollet \& Little, 2017). Eleven participants were excluded for being ambidextrous due to the possibility their left hand is more dominant $\left(n_{\text {Final }}=274 ; 237\right.$ women, 35 men, 2 unreported; $M_{\text {Age }}=21.10, S D=4.99 ; 52.6 \%$ White, $39.2 \%$ Black, 4.4\% Latin, 1.8\% Asian, 1.8\% Other).

\subsubsection{Materials and procedure}

Participants were first primed with competition $(n=144)$ or cooperation $(n=130)$ like in Study 3. They then completed the line bisection task by judging whether horizontal lines were longer on the left or right side of a perpendicular line to measure visual field bias, a technique to assess neural activity that underpins approach/avoidance motives (Miller et al., 2012). Due to contralateral connections in cortical areas implicated in motivations (Nash, McGregor, \& Inzlicht, 2010), right cortical activity is associated with avoidance responses toward aversive stimuli that elicit left visual field biases. Left cortical activity is associated with approach responses toward appetitive stimuli that elicit right visual field biases.

Participants viewed 10 filler trials with asymmetrically bisected lines and 20 critical trials with centrally bisected lines in random order. Above each line was a face (Slepian et al., 2017). Critical trials displayed high- and low-fWHR targets. We coded indication of centrally bisected lines as longer on the left (LVF-bias) as 0 and longer on the right as 1 (RVF-bias). We calculated proportions for high- and lowfWHR targets separately by summing responses of each trial divided by number of trials. Scores below 0.5 indicated LVF-bias. Scores above 0.5 RVF-bias.

\subsection{Results}

We used a 2 (condition: competition vs. cooperation) $\times 2$ (target fWHR: high vs. low) mixed-model ANOVA with repeated factors over target fWHR. A target fWHR main effect indicated participants reported more LVF-bias toward high-fWHR targets $(M=0.44, S D=0.22)$ than low-fWHR targets $(M=0.47, S D=0.22), F(1,272)=4.77$, $p=0.030, \eta_{p}{ }^{2}=0.017$. Neither the Condition main effect nor interaction emerged, $F \mathrm{~s}<0.07, \mathrm{ps}>0.805$.

Regarding whether these LVF-biases were categorical, we conducted one-sample $t$-tests weighted against the midpoint of 0.5 (i.e., no VF-bias). Participants reported a categorical LVF-bias for high-fWHR targets suggesting activation of avoidance motives, $t(273)=3.97$, $p<0.001, d=0.24$. The low-fWHR target bias was not categorical, $t$ (273) $=1.74, p=0.083, d=0.10$.

\subsection{Discussion}

High-fWHR targets elicited aversion, possibly reflecting perceptions of high-fWHR men as threatening. Bias against high-fWHR men could be difficult to override, given perceptions of exploitativeness. The preferences for high-fWHR men in previous studies in this paper could reflect more of a tolerance than an interest (see Brown, Sacco, Lolley, \& Block, 2017; Brown, Tracy, Young, \& Sacco, 2021). Alternatively, perceptions of high-fWHR men as threatening could be resistant to motivational priming at the automatic level. The nuance in formidability assessments may only be discernable through conscious assessments, wherein perceivers override automatic associations through effortful judgments to recognize coalitional benefits despite the salient costs.

\section{General discussion}

Five experiments indicated that affordance judgments of formidability, inferred through men's fWHR, were the basis of individuals' preference for formidability in tasks requiring strength. We further found that competitive motives fostered preferences for high-fWHR men, though this preference was ultimately rooted in a tolerance for formidable men. Results contribute to growing research demonstrating how formidable facial features, particularly fWHR, influence preferences for cooperative alliances to increase access to resources and security despite the potential risks of aggressive men (Barbaro, Mogilski, Shackelford, \& Pham, 2018; Brown et al., 2017).

Although findings continue to demonstrate associations between perceived formidability and the presence of masculinized features, fWHR is unreliably associated with other trait inferences across cultures, such as dominance (Durkee \& Ayers, 2021). This unreliability of certain trait inferences highlights the likelihood of specific ratios in human bodies not actually being selected so much as the individual components comprising these ratios (Richardson, 2020). Selection for highfWHR men could be pleiotropic: Desirable features within the ratio were sexually selected for a benefit, with covarying traits being indirectly selected (Dixson, 2018). The perceived formidability of highfWHR men could prompt them to recognize their advantage in conflict and foster aggression via self-fulfilling prophecies (Haselhuhn et al., 2013).

Identifying underpinnings of fWHR in sexual selection makes it crucial to discuss specificity. Conflicting findings exist for whether fWHR is reliably associated with trait inferences, including dominance or aggression, for example. Archival and machine learning studies indicate that fWHR is uninformative in many inferences, particularly in nonWestern cultures (Durkee \& Ayers, 2021; Jaeger \& Jones, n.d.). Inconsistencies should motivate conversations on the robustness of various fWHR affordance judgments and trait inferences. Aggression in male faces is consistently inferred cross-culturally, suggesting that research would benefit from focusing on physical capabilities and emotional states rather personality traits.

\subsection{Limitations and future directions}

Several limitations emerged, which require continued research to address. Although we identified contexts under which high- and lowfWHR influence preferences and affordance judgments, it is likely that additional contextual factors influence whether perceivers prefer or avoid high-fWHR men. Increased salience of threats to one's physical safety heightens preference for formidable men, which could serve to protect the perceiver from danger (Brown, Donahoe, \& Boykin, 2022; Sacco, Lustgraaf, Brown, \& Young, 2015), though chronic environmental danger is frequently associated with an aversion to dominant faces (Borras-Guevara et al., 2017; Brooks et al., 2011). Future research could identify how motivational states shift valuations of high-fWHR.

Preferences for high-fWHR men were based on heuristic judgments and not actual formidability. Future research would benefit from assessing actual formidability of prospective allies, perhaps through handgrip strength or win-loss records in competitions (e.g., Caton, Hannan, \& Dixson, 2022; Zilioli et al., 2015). A study could assess coalitional affordances of MMA fighters while identifying features from which perceivers make decisions beyond the heuristics of fWHR.

Our exclusively U.S. samples necessitate future research to fWHR judgments cross-culturally. Dominance inferences appear less universal than previously thought (Jones et al., 2021). Nonetheless, formidability remains accurately inferred through components of fWHR in Western and non-Western societies (Butovskaya et al., 2018; Zilioli et al., 2015). Amazonian societies perceive high-fWHR men as effective hunters, yet ineffective warriors, a difference possibly reflecting cultural conceptualizations of traits (Magginetti, 2015). Future research would benefit 
from identifying cross-cultural differences in coalition preferences. Sexually selected components of fWHR are also sexually dimorphic in both WEIRD and non-WEIRD societies (Hodges-Simeon et al., 2021). Understanding the consistency of cross-cultural findings would facilitate understanding the affordances connoted through fWHR.

\section{Conclusion}

The current studies provided robust evidence indicating that highfWHR men are perceived as especially formidable and therefore most desirable for group tasks that require physical formidability. The preference for high-fWHR men was limited to domains related to strength and protection, potentially related to the inferred costs of formidability, such as low intelligence or aggressiveness. Individuals appear to weigh the costs and benefits associated with affordances of formidable and non-formidable men when determining how well they can facilitate group goals.

\section{Acknowledgement}

We thank Steven Young and Nicholas Brown for their helpful commentary and feedback on earlier version of this manuscript.

\section{References}

Apicella, C.L. (2014). Upper-body strength predicts hunting reputation and reproductive success in Hadza hunter-gatherers. Evolution and Human Behavior, 35, 508-518.

Aung, T., Rosenfield, K.A., \& Puts, D. (2021). Male voice pitch mediates the relationship between objective and perceived formidability. Evolution and Human Behavior, 42, 121-129.

Barbaro, N., Mogilski, J.K., Shackelford, T.K., \& Pham, M.N. (2018). Men's interest in allying with a previous combatant for future group combat. Human Nature, 29, 328-336.

Borras-Guevara, M.L., Batres, C., \& Perrett, D.I. (2017). Aggressor or protector? Experiences and perceptions of violence predict preferences for masculinity. Evolution and Human Behavior, 38, 481-489.

Brooks, R., Scott, I.M., Maklakov, A.A., Kasumovic, M.M., Clark, A.P., \& Penton-Voak, I.S. (2011). National income inequality predicts women's preferences for masculinized faces better than health does. Proceedings of the Royal Society B: Biological Sciences, 278, 810-812.

Brown, M., Bauer, B.W., Sacco, D.F., \& Capron, D.W. (2021). Functional inferences of formidability bias perceptions of mental distress. Evolutionary Psychological Science, 7, $401-410$.

Brown, M., Donahoe, S., \& Boykin, K. (2022). Physical strength as a cue to men's capability as protective parents. Evolutionary Psychological Science, 8, 81-88.

Brown, M., Sacco, D.F., \& Drea, K.M. (2022). Ecologically contingent preferences for formidable coalitional allies as a function of conservative ideologies. Personality and Individual Differences, 195, 111699.

Brown, M., Sacco, D.F., Lolley, K.P., \& Block, D. (2017). Facing the implications: Dangerous world beliefs differentially predict men and women's aversion to facially communicated psychopathy. Personality and Individual Differences, 116, 1-5.

Brown, M., Sacco, D.F., \& Medlin, M.M. (2019). Women's short-term mating goals elicit avoidance of faces whose eyes lack limbal rings. Evolutionary Behavioral Sciences, 13, $278-285$.

Brown, M., Tracy, R.E., \& Boykin, K. (2022). Covariation between formidability inferences and perceptions of men's humor styles. Personality and Individual Differences. In press.

Brown, M., Tracy, R.E., Young, S.G., \& Sacco, D.F. (2021). Crowd salience heightens tolerance to healthy facial features. Adaptive Human Behavior and Physiology, 7, 432-446.

Butovskaya, M.L., Windhager, S., Karelin, D., Mezentseva, A., Schaefer, K., \& Fink, B. (2018). Associations of physical strength with facial shape in an African pastoralist society, the Maasai of Northern Tanzania. PLoS One, 13, e0197738.

Carré, J.M., McCormick, C.M., \& Mondloch, C.J. (2009). Facial structure is a reliable cue of aggressive behavior. Psychological Science, 20, 1194-1198.

Caton, N.R., \& Dixson, B.J.W. (2022). Beyond facial width-to-height ratios: Bizygomatic width is highly sexually dimorphic when adjusting for allometry. Preprint available PsyArxiv.

Brown, M., Sacco, D. F., \& Barbaro, N. (2022). Formidable facial structures influence postconflict reconciliation judgments. Evolutionary Behavioral Sciences, Online-first publication.

Caton, N. R., Hannan, J., \& Dixson, B. J. (2022). Facial width-to-height ratio predicts fighting success: A direct replication and extension of Zilioli et al. (2014). Aggressive Behavior, Online-first publication.

Caton, N.R., Zhao, A., Lewis, D.M.G., \& Dixson, B.J.W. (2022). Facial masculinity predicts men's actual and perceived aggressiveness. Preprint available PsyArxiv.

Christiansen, K., \& Winkler, E.M. (1992). Hormonal, anthropometrical, and behavioral correlates of physical aggression in! Kung san men of Namibia. Aggressive Behavior, 18, 271-280.

Deaner, R.O., Goetz, S.M., Shattuck, K., \& Schnotala, T. (2012). Body weight, not facial width-to-height ratio, predicts aggression in pro hockey players. Journal of Research in Personality, 46, 235-238.

DeBruine, L.M., Jones, B.C., Little, A.C., Crawford, J.R., \& Welling, L.L. (2011). Further evidence for regional variation in women's masculinity preferences. Proceedings of the Royal Society B: Biological Sciences, 278, 813-814.

Caton, N. R., Pearson, S. G., \& Dixson, B. J. (2022). Is facial structure an honest cue to realworld dominance and fighting ability in men? A pre-registered direct replication of. Evolution and Human Behavior, Online-first publication.

Deska, J. C., Hingston, S. T., DelVecchio, D., Stenstrom, E. P., Walker, R. J., \& Hugenberg, K. (n.d.). The face of the brand: Spokesperson facial width-to-height ratio predicts brand personality judgments. Psychology and Marketing, (in press).

Deska, J.C., \& Hugenberg, K. (2018). Targets' facial width-to-height ratio biases pain judgments. Journal of Experimental Social Psychology, 74, 56-64.

Deska, J.C., Lloyd, E.P., \& Hugenberg, K. (2018a). The face of fear and anger: Facial width to-height ratio biases recognition of angry and fearful expressions. Emotion, 18, 453-464.

Deska, J.C., Lloyd, E.P., \& Hugenberg, K. (2018b). Facing humanness: Facial width-toheight ratio predicts ascriptions of humanity. Journal of Personality and Social Psychology, 114, 75-94.

Dixson, B.J. (2018). Is male facial width-to-height ratio the target of sexual selection? Archives of Sexual Behavior, 47, 827-828.

Dixson, B.J., Lee, A.J., Sherlock, J.M., \& Talamas, S.N. (2017). Beneath the beard: Do facial morphometrics influence the strength of judgments of men's beardedness? Evolution and Human Behavior, 38, 164-174.

Durkee, P.K., \& Ayers, J.D. (2021). Is facial width-to-height ratio reliably associated with social inferences? Evolution and Human Behavior, 42, 583-592.

Geniole, S.N., Denson, T.F., Dixson, B.J., Carré, J.M., \& McCormick, C.M. (2015). Evidence from meta-analyses of the facial width-to-height ratio as an evolved cue of threat. PLoS One, 10, e0132726.

Geniole, S.N., \& McCormick, C.M. (2013). Taking control of aggression: Perceptions of aggression suppress the link between perceptions of facial masculinity and attractiveness. Evolutionary Psychology, 11, 1027-1043.

Geniole, S.N., \& McCormick, C.M. (2015). Facing our ancestors: Judgements of aggression are consistent and related to the facial width-to-height ratio in men irrespective of beards. Evolution and Human Behavior, 36, 279-285.

Goetz, S.M., Shattuck, K.S., Miller, R.M., Campbell, J.A., Lozoya, E., Weisfeld, G.E., \& Carré, J.M. (2013). Social status moderates the relationship between facial structure and aggression. Psychological Science, 24, 2329-2334.

Griggs, R.C., Kingston, W., Jozefowicz, R.F., Herr, B.E., Forbes, G., \& Halliday, D. (1989). Effect of testosterone on muscle mass and muscle protein synthesis. Journal of Applied Physiology, 66, 498-503.

Harmon-Jones, E., \& Allen, J.J. (1998). Anger and frontal brain activity: EEG asymmetry consistent with approach motivation despite negative affective valence. Journal of Personality and Social Psychology, 74, 1310-1316.

Haselhuhn, M.P., Ormiston, M.E., \& Wong, E.M. (2015). Men's facial width-to-height ratio predicts aggression: A meta-analysis. PLoS One, 10, e0122637.

Haselhuhn, M.P., Wong, E.M., \& Ormiston, M.E. (2013). Self-fulfilling prophecies as a link between men's facial width-to-height ratio and behavior. PLoS One, 8, e72259.

Hayes, A.F. (2013). Introduction to mediation, moderation, and conditional process analysis: A regression-based approach. Guilford Publications.

Hehman, E., Leitner, J.B., Deegan, M.P., \& Gaertner, S.L. (2015). Picking teams: When dominant facial structure is preferred. Journal of Experimental Social Psychology, 59, $51-59$.

Hehman, E., Leitner, J.B., \& Gaertner, S.L. (2013). Enhancing static facial features increases intimidation. Journal of Experimental Social Psychology, 49, 747-754.

Hodges-Simeon, C.R., Albert, G., Richardson, G.B., McHale, T.S., Weinberg, S.M., Gurven, M., \& Gaulin, S.J. (2021). Was facial width-to-height ratio subject to sexual selection pressures? A life course approach. PLoS One, 16, e0240284.

Hodges-Simeon, C.R., Hanson Sobraske, K.N., Samore, T., Gurven, M., \& Gaulin, S.J. (2016). Facial width-to-height ratio (fWHR) is not associated with adolescent testosterone levels. PLoS One, 11, e0153083.

Holzleitner, I.J., \& Perrett, D.I. (2016). Perception of strength from 3D faces is linked to facial cues of physique. Evolution and Human Behavior, 37, 217-229.

Jaeger, B., \& Jones, A. (n.d.). Which facial features are central in impression formation? Social Psychological and Personality Science, In press.

Jewell, G., \& McCourt, M.E. (2000). Pseudoneglect: A review and meta-analysis of performance factors in line bisection tasks. Neuropsychologia, 38, 93-110.

Jones, B.C., DeBruine, L.M., Flake, J.K., Liuzza, M.T., Antfolk, J., Arinze, N.C., Sirota, M. (2021). To which world regions does the valence-dominance model of social perception apply? Nature Human Behaviour, 5, 159-169.

Kajonius, P., \& Eldblom, H. (2020). Facial width-to-height ratio as a cue of threat: An initial event-related potential study. International Journal of Psychological and Brain Sciences, 5, $1-4$.

Kosinski, M. (2017). Facial width-to-height ratio does not predict self-reported behavioral tendencies. Psychological Science, 28, 1675-1682.

Lefevre, C.E., Lewis, G.J., Bates, T.C., Dzhelyova, M., Coetzee, V., Deary, I.J., \& Perrett, D.I. (2012). No evidence for sexual dimorphism of facial width-to-height ratio in four large adult samples. Evolution and Human Behavior, 33, 623-627.

Lieberz, K.A., Windmann, S., Geniole, S.N., McCormick, C.M., Mueller-Engelmann, M., Gruener, F., ... Steil, R. (2017). The facial width-to-height ratio determines interpersonal distance preferences in the observer. Aggressive Behavior, 43, 460-470.

Liu, L., Wen, G., \& Zheng, L. (2022). Facial width to height ratio and perceived aggression: The disjunction effect of horizontal and vertical components. Personality and Individual Differences, 191, 111578.

Lukaszewski, A.W., Simmons, Z.L., Anderson, C., \& Roney, J.R. (2016). The role of physical formidability in human social status allocation. Journal of Personality and Social Psychology, 110, 385-406. 
Ma, D.S., Correll, J., \& Wittenbrink, B. (2015). The Chicago face database: A free stimulus set of faces and norming data. Behavior Research Methods, 47, 1122-1135.

MacDonell, E.T., Geniole, S.N., \& McCormick, C.M. (2018). Force versus fury: Sex differences in the relationships among physical and psychological threat potential, the facial width-to-height ratio, and judgements of aggressiveness. Aggressive Behavior, 44, 512-523.

Magginetti, G. (2015). Facial width-to-height ratio and attraction in a small scale Amazonian society. Unpublished Master's Thesis.

Matsumoto, D., \& Hwang, H.C. (2021). Facial width-to-height ratios and deception skill. Personality and Individual Differences, 174, 110683.

McDonald, M.M., Navarrete, C.D., \& Van Vugt, M. (2012). Evolution and the psychology of intergroup conflict: The male warrior hypothesis. Philosophical Transactions of the Royal Society, B: Biological Sciences, 367, 670-679.

Mileva, V.R., Cowan, M.L., Cobey, K.D., Knowles, K.K., \& Little, A.C. (2014). In the face of dominance: Self-perceived and other-perceived dominance are positively associated with facial-width-to-height ratio in men. Personality and Individual Differences, 69, 115-118.

Miller, S.L., Prokosch, M.L., \& Maner, J.K. (2012). Relationship maintenance and biases on the line bisection task: Attractive alternatives, asymmetrical cortical activity, and approach-avoidance motivation. Journal of Experimental Social Psychology, 48, 566-569.

Montoya, R.M., \& Pittinsky, T.L. (2013). Individual variability in adherence to the norm of group interest predicts outgroup bias. Group Processes \& Intergroup Relations, 16, 173-191.

Nash, K., McGregor, I., \& Inzlicht, M. (2010). Line bisection as a neural marker of approach motivation. Psychophysiology, 47, 979-983.

Neuberg, S.L., Kenrick, D.T., \& Schaller, M. (2011). Human threat management systems: Self-protection and disease avoidance. Neuroscience \& Biobehavioral Reviews, 35, 1042-1051.

Phillips, L.T., Slepian, M.L., \& Hughes, B.L. (2018). Perceiving groups: The people perception of diversity and hierarchy. Journal of Personality and Social Psychology, 114, $766-785$.

Pollet, T., \& Little, A. (2017). Baseline probabilities for two-alternative forced choice tasks when judging stimuli in evolutionary psychology: A methodological note. Human Ethology Bulletin, 32, 53-59.

Price, M.E., Sheehy-Skeffington, J., Sidanius, J., \& Pound, N. (2017). Is sociopolitical egalitarianism related to bodily and facial formidability in men? Evolution and Human Behavior, 38, 626-634.

Puts, D.A. (2010). Beauty and the beast: Mechanisms of sexual selection in humans. Evolution and Human Behavior, 31, 157-175.

Richardson, T. (2020). Ratios in the evolutionary behavioural sciences: Problems and solutions. Preprint available via PsyArxiv.

von Rueden, C., \& Van Vugt, M. (2015). Leadership in small-scale societies: Some implications for theory, research, and practice. The Leadership Quarterly, 26, 978-990.
Sacco, D.F., Lustgraaf, C.N.J., Brown, M., \& Young, S.G. (2015). Activation of selfprotection threat increases women's preferences for dominance in male faces. Human Ethology Bulletin, 30, 24-32.

Sell, A., Hone, L.S., \& Pound, N. (2012). The importance of physical strength to human males. Human Nature, 23, 30-44.

Sell, A., Tooby, J., \& Cosmides, L. (2009). Formidability and the logic of human anger. Proceedings of the National Academy of Sciences, 106, 15073-15078.

Slepian, M.L., Young, S.G., \& Harmon-Jones, E. (2017). An approach-avoidance motivational model of trustworthiness judgments. Motivation Science, 3, 91-97.

Snyder, J.K., Fessler, D.M., Tiokhin, L., Frederick, D.A., Lee, S.W., \& Navarrete, C.D. (2011). Trade-offs in a dangerous world: Women's fear of crime predicts preferences for aggressive and formidable mates. Evolution and Human Behavior, 32, 127-137.

Stirrat, M., Stulp, G., \& Pollet, T.V. (2012). Male facial width is associated with death by contact violence: Narrow-faced males are more likely to die from contact violence. Evolution and Human Behavior, 33, 551-556.

Thomas, D.R., \& Zumbo, B.D. (2012). Difference scores from the point of view of reliability and repeated-measures ANOVA: In defense of difference scores for data analysis. Educational and Psychological Measurement, 72, 37-43.

Toscano, H., Schubert, T.W., \& Giessner, S.R. (2018). Eye gaze and head posture jointly influence judgments of dominance, physical strength, and anger. Journal of Nonverbal Behavior, 42, 285-309.

Trafimow, D. (2015). A defense against the alleged unreliability of difference scores. Cogent Mathematics, 2, 1064626.

Třebický, V., Fialová, J., Kleisner, K., Roberts, S.C., Little, A.C., \& Havlíček, J. (2015). Further evidence for links between facial width-to-height ratio and fighting success: Commentary on Zilioli et al. (2014). Aggressive Behavior, 41, 331-334.

Welker, K.M., Goetz, S.M., Galicia, S., Liphardt, J., \& Carré, J.M. (2015). An examination of the associations between facial structure, aggressive behavior, and performance in the 2010 world cup association football players. Adaptive Human Behavior and Physiology, 1, $17-29$.

Whitehouse, A.J., Gilani, S.Z., Shafait, F., Mian, A., Tan, D.W., Maybery, M.T., ... Eastwood, P. (2015). Prenatal testosterone exposure is related to sexually dimorphic facial morphology in adulthood. Proceedings of the Royal Society B: Biological Sciences, $282,20151351$.

Zebrowitz, L.A., \& Montepare, J. (2006). The ecological approach to person perception: Evolutionary roots and contemporary offshoots.

Zilioli, S., Sell, A.N., Stirrat, M., Jagore, J., Vickerman, W., \& Watson, N.V. (2015). Face of a fighter: Bizygomatic width as a cue of formidability. Aggressive Behavior, 41, $322-330$. 